\title{
SEARCH FOR HIGGS BOSONS WITH THE ATLAS DETECTOR
}

\author{
Simonetta Gentile ${ }^{a}$ \\ Università di Roma,"La Sapienza", Sezione di Roma1 dell'INFN, Roma, Italy \\ Abstract. The Large Hadron Collider experiments are designed to fully explore \\ new physics at the $\mathrm{TeV}$ center-of-mass region. The performance and the discovery \\ potential of the LHC detectors, in particularly of ATLAS, for the only unobserved \\ Standard Model particle, the Higgs boson, are discussed. The discussion includes \\ also the supersymmetric Higgs bosons, postulated in the Minimal Supersymmetric \\ extension to the Standard Model.
}

\section{Introduction}

For one decade, precision tests of the Standard Model of Electroweak Interactions (SM) [1] have been performed at LEP. The observed consistency provided great confidence in the validity of the SM and encouraged the use of all experimental data to predict the mass of the last unobserved particle of the model: the Higgs boson. The derived limit on its mass from electroweak fit is $\mathrm{m}_{\mathrm{H}}<219 \mathrm{GeV}$ at $95 \%$ Confidence Level [2]. Direct searches for the Standard Model Higgs set a lower limit on its mass $\mathrm{m}_{\mathrm{H}}>114.4 \mathrm{GeV}$ at $95 \%$ Confidence Level [3].

Despite the success of the precision tests of the SM, extensions of the Standard Model have been proposed, of which the most investigated is the Minimal Supersymmetric Standard Model (MSSM). This theory requires two Higgs doublets. This gives rise to five Higgs bosons: a charged scalar pair, two neutral $\mathrm{CP}$-even, the lightest of which is called $\mathrm{h}$, and a neutral CP-odd, A [4]. The discovery of any one of these particles is crucial to prove the validity of the model. Based on LEP data, limits on the masses of charged, $\mathrm{H}^{ \pm}[5]$, and neutral Higgs bosons, $\mathrm{h}$ and $\mathrm{A}[6]$, exist over a large region of the parameter space.

Recent studies by the Tevatron experiments show a reduction in the amount of integrated luminosity needed to discover Higgs bosons at the Tevatron collider relative to previous studies. These improvements come from more optimized methods of analysis. An integrated luminosity of $4-8 \mathrm{fb}^{-1}$, expected to be collected by 2009 , is needed for a $95 \%$ C.L. exclusion of a Higgs boson with $m_{\mathrm{H}} \approx 120 \mathrm{GeV}$ [7].

One of the goals of the Large Hadron Collider (LHC) general-purpose experiments, ATLAS and CMS, is to look for the SM Higgs boson and to prove or exclude the existence of supersymmetric Higgs bosons in the parameters region not excluded by the LEP data. This ambitious program requires high performance detectors and high luminosity from the machine.

This article is organized as follows: Section 2 reviews the expected performance of one of the general-purpose detectors, ATLAS, and expected integrated

\footnotetext{
${ }^{a}$ e-mail: simonetta.gentile@cern.ch,simonetta.gentile@roma1.infn.it
} 
luminosities. The production of the SM Higgs in pp interactions at $14 \mathrm{TeV}$, its decay modes and the expected backgrounds are described in Section 3. The discovery potential for various integrated luminosity scenarios are discussed in Section 4 and the measurement of the Higgs properties, such as mass and couplings, in Section 5. The hunt of supersymmetric Higgs is reviewed in Section 6. The conclusions and future prospects for the future are given is Section 7 .

\section{Experimental tools: Large Hadron Collider, ATLAS.}

The Large Hadron Collider, now under construction at CERN, will collide proton beams at centre-of-mass energy of $14 \mathrm{TeV}$, with very high luminosity $\left(10^{33}-10^{34} \mathrm{~cm}^{-2} \mathrm{~s}^{-1}\right)$. The first physics data will be collected in 2007. In the following the main features of the ATLAS detector are briefly summarized [9]:

- Superconducting Magnet: It is composed of: a) a solenoid, which provides a $2 \mathrm{~T}$ magnetic field for the inner detector, b) a barrel air toroid, consisting of eight flat coils of $25 \mathrm{~m}$ length. The bending power for muons is typically $4 \mathrm{~T} \cdot \mathrm{m}$ and c) Two end-cap toroids, which provide a field of $6 \mathrm{~T} \cdot \mathrm{m}$ for muon momentum measurements.

- Inner Tracking detector: This is inside in the solenoid magnetic field and consists of 140 million $\mathrm{Si}$ pixels and 6 million silicon strip detectors near the interaction point, and 0.4 million strawtubes. The momentum resolution of the inner tracker for charged particle is $\frac{\sigma}{\mathrm{pT}} \sim$ $5 \times 10^{-4} \mathrm{p}_{\mathrm{T}}(\mathrm{GeV}) \oplus 0.01$.

- Electromagnetic Calorimeter: This is a liquid argon-lead sampling calorimeter with accordion shape in the barrel and end-cap regions (total of about 180000 channels). The energy resolution is $\frac{\sigma}{\mathrm{E}} \sim \frac{10 \%}{\sqrt{\mathrm{E}}}$.

- Hadronic Calorimeter: It is a copper-liquid argon calorimeter in the end-cap region ( 10,000 channels) and a Fe-scintillator calorimeter scintillators ( 10,000 channels) in barrel region. The liquid argon tungsten forward calorimeters extend the coverage to $|\eta|=4.9$. The hadronic jet energy resolution is $\frac{\sigma}{\mathrm{E}} \sim \frac{50 \%}{\sqrt{\mathrm{E}}} \oplus 0.03$.

- Muon spectrometer: a) 1194 precision tracking chambers made of more than $3.710^{6}$ Monitored Drift Tubes (MDT) covering the rapidity range $|\eta|<2.7$. The momentum resolution ranges from about $2 \%$ for 100 GeV muons to $10 \%$ for $1 \mathrm{TeV}$ muons. b) 32 Cathode Strip Chambers (CSC) consisting of more than 24 thousand precision and 6 thousand transverse coordinate strips covering the most forward rapidity region $(|\eta|=2.0-2.7)$ in the inner most stations of the muon system. c) 596 Resistive Plate Chambers (RPC) in the barrel region $(|\eta|<1)$ and 
4256 Thin Gap Chambers (TGC) in the end-cap region to provide muon triggers and to measure the second coordinate of the muon tracks.

The ATLAS detector will have excellent lepton and photon identification capabilities, and accurate energy and angular measurements over almost $4 \pi$ coverage.

The expected potential will be discussed under different assumptions on the integrated luminosity:

- $\int \mathcal{L} \mathrm{dt}=10 \mathrm{fb}^{-1}$, corresponding to data collected in approximately one year of data taking at the initial LHC luminosity $\left(\mathcal{L}=10^{33} \mathrm{~cm}^{-2} \mathrm{~s}^{-1}\right)$.

- $\int \mathcal{L} \mathrm{dt}=30 \mathrm{fb}^{-1}$, collected in 3 years at low luminosity.

- $\int \mathcal{L} \mathrm{dt}=100 \mathrm{fb}^{-1}$, collected in one year at high luminosity,

- Ultimate luminosity $\int \mathcal{L} \mathrm{dt}=300 \mathrm{fb}^{-1}$.

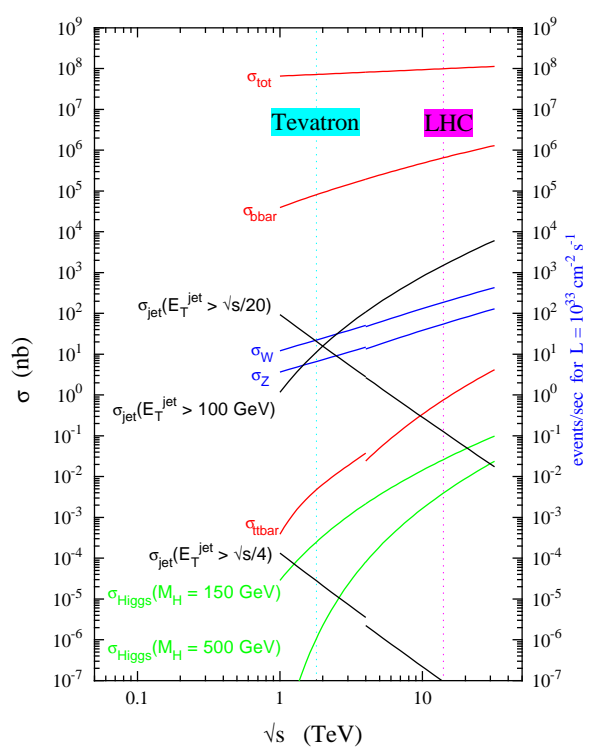

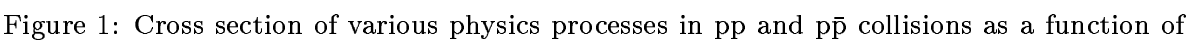
center-of-mass energy.

\section{Standard Model Higgs: production and decays.}

The search for the Standard Model Higgs has been studied in ATLAS looking at a large number of different production and decay modes up $1 \mathrm{TeV}$ [9]. The 
difficulty to extract the Higgs signal from the huge QCD background dictates the choice of channels with leptons and photons. Fig. 1 shows that the crosssection of the QCD backgrounds is many orders of magnitude higher than the cross-section of a Higgs of mass $150 \mathrm{GeV}$. The most copious background is made of $\mathrm{QCD}$ jets and $\mathrm{W}, \mathrm{Z}$ gauge boson production. At the LHC the production

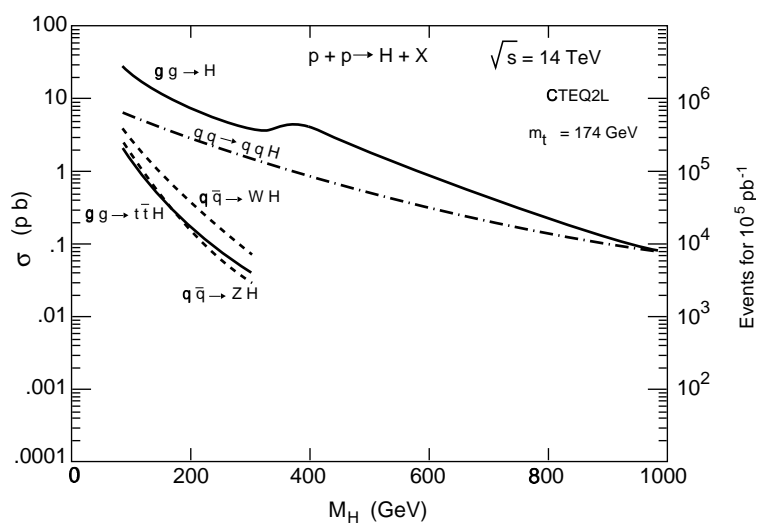

Figure 2: Higgs production cross section at LHC as a function of $m_{\mathrm{H}}$ for different production mechanism.

cross section for the Standard Model Higgs boson is dominated by gluon-gluon fusion (Fig.2); the second most important process is the fusion of vector bosons radiated from initial-state quarks. The relative contributions of the two processes depend on the Higgs boson mass. For $m_{\mathrm{H}}<2 m_{\mathrm{Z}}$, vector boson fusion amounts to about $20 \%$ of the total production cross section, and becomes more important with increasing mass. Other production mechanisms, such as associated production $\mathrm{ttH}, \mathrm{WH}, \mathrm{ZH}$, have cross-sections of approximately $1-10 \%$ of the gluon-gluon fusion. Fig.3 shows the Higgs decay modes as a function of $m_{\mathrm{H}}$. The dominant decay modes are $\mathrm{W}^{+} \mathrm{W}^{-}$and $\mathrm{ZZ}$ for Higgs mass $m_{\mathrm{H}}>2 m_{\mathrm{Z}}$ and $\mathrm{H} \rightarrow \mathrm{b} \overline{\mathrm{b}}$ for $m_{\mathrm{H}}<2 m_{\mathrm{Z}}$. Because of the large QCD background it is necessary to rely on Higgs decays that produce isolated energetic leptons or photons in the final state.

The Higgs discovery criterion used here is the so called " $5 \sigma$ signal significance", defined as $\frac{S}{\sqrt{B}} \geq 5$, where $\mathrm{S}$ is the number of signal events and B the number of background events.

Recent analyses have shown the promising contribution of WW fusion in the low-mass range [10]. In this process the Higgs boson is accompanied by two jets in the forward regions of the detector, originating from the initial quarks that emit the vector bosons. In addition, central jet activity is suppressed due the lack of color exchange between the quarks. This is in contrast to most background processes, where there is color flow in the t-channel. Therefore jet 


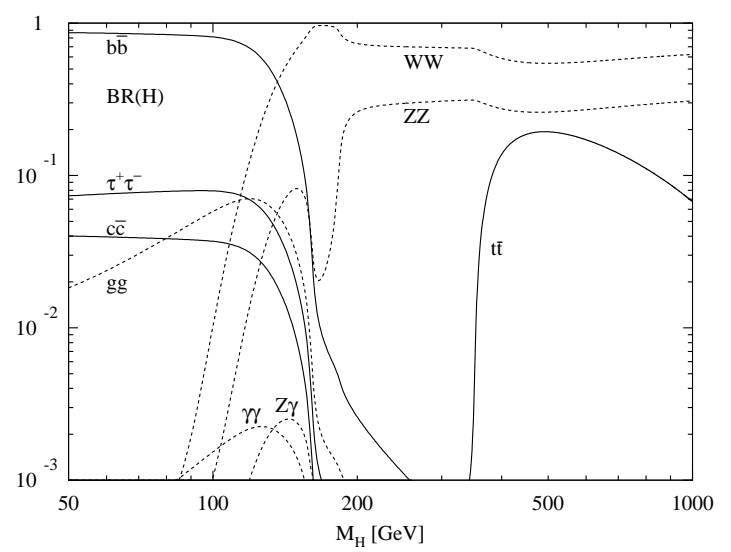

Figure 3: Higgs decay branching ratios as a function of $m_{\mathrm{H}}$. The mass region excluded by LEP direct searches is below $114 \mathrm{GeV}$.

tagging in the forward region of the detector, with a veto of jet activity in the central region, are useful tools to enhance the signal-to-background ratio.

\section{Standard Model Higgs boson discovery potential.}

The LEP data favour the $m_{\mathrm{H}}$ region between $114.4 \mathrm{GeV}$ and $219 \mathrm{GeV}$. In this region a combined $5 \sigma$ significance can be reached by combining the decays $\mathrm{ttH} \rightarrow \mathrm{ttb} \overline{\mathrm{b}}, \mathrm{H} \rightarrow \gamma \gamma, \mathrm{H} \rightarrow \mathrm{WW}^{(*)}, \mathrm{H} \rightarrow \mathrm{ZZ}^{(*)}$. In the low mass region $\left(m_{\mathrm{H}}<130 \mathrm{GeV}\right)$ the Higgs will be searched mainly through $\mathrm{H} \rightarrow \gamma \gamma$ and the associated production ttH. Exploiting the characteristics of the WW-fusion channel it is possible to extract a very clear signal from $\mathrm{H} \rightarrow \mathrm{WW}^{*} \rightarrow \ell \nu \ell \nu$. The Higgs is produced with a pseudorapidity distribution $\Delta \eta$ of the tag jets, which allows a good separation of signal from background (Fig. 4a) . Including all channels, each experiment should be able to discover a SM Higgs with only $10 \mathrm{fb}^{-1}$, expected to be collected in one year of data taking (Fig. 5).

In this region the observation of all channels is important to reach the 4$5 \sigma$ significance. For heavy Higgs mass $\left(m_{\mathrm{H}}>2 m_{\mathrm{Z}}\right)$ the search will be easier through the $\mathrm{H} \rightarrow \mathrm{ZZ} \rightarrow 4 \ell$ gold-plated channel(Fig $4 \mathrm{~b})$.

\subsection{Standard Model Higgs: Parameter measurements}

Although the Higgs boson discovery is possible with only $\int \mathcal{L} \mathrm{dt}=10 \mathrm{fb}^{-1}$, higher integrated luminosity is necessary to measure the Higgs parameters, e.g. mass, width, branching ratios and couplings. The ultimate luminosity, $\int \mathcal{L} \mathrm{dt}=300 \mathrm{fb}^{-1}$, is necessary to measure the Higgs mass with a precision of $0.1 \%$ over the mass range between 130 and $450 \mathrm{GeV}$ [11].

Recent results for Higgs-coupling measurements with a global fit method use all the production modes and detection channels with the ultimate luminosity. 

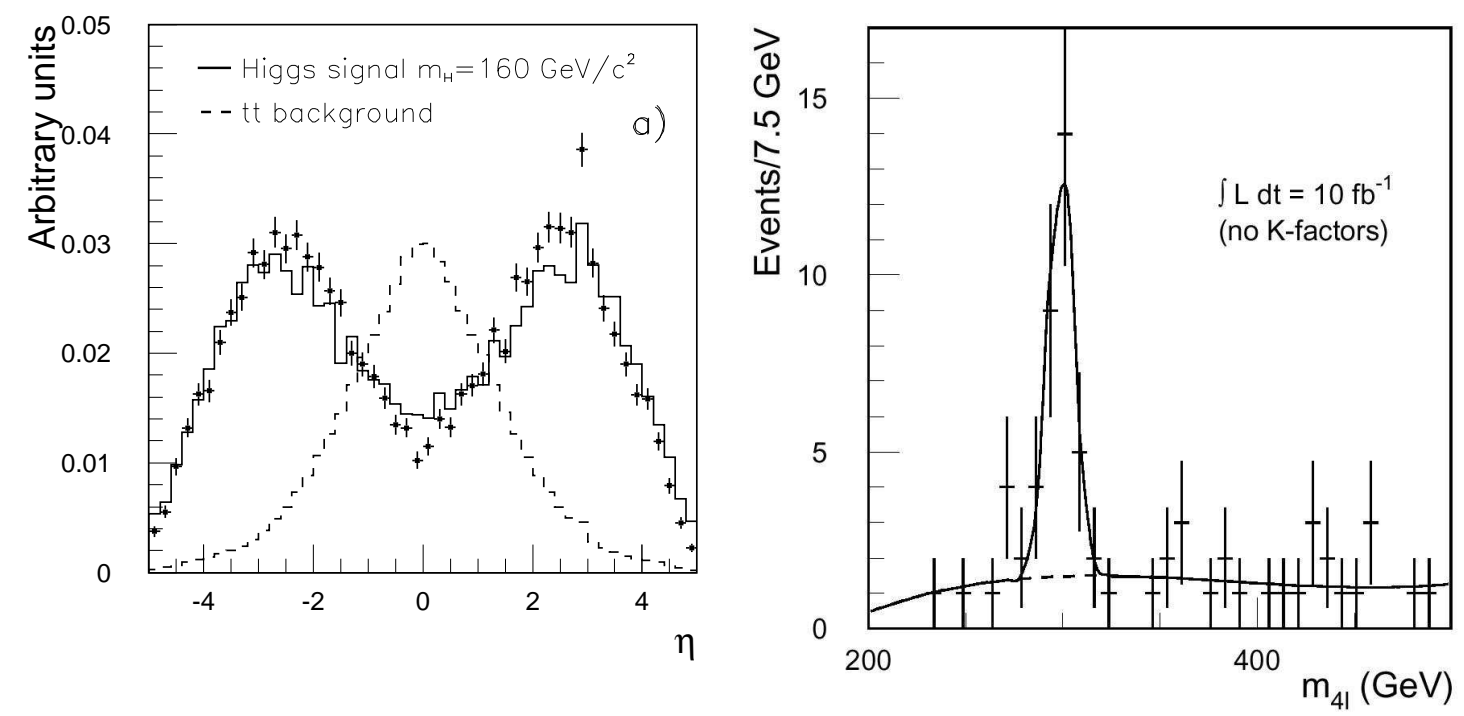

Figure 4: a) Pseudorapidity distribution of the tag jets in qqH events with $m_{\mathrm{H}}=160 \mathrm{GeV}$ and in the tt background (left). b) Invariant mass distribution for $300 \mathrm{GeV}$ Higgs signal over background in the four lepton final state for $\int \mathcal{L} \mathrm{dt}=10 \mathrm{fb}^{-1}$ (right) .

The branching ratio $\mathrm{H} \rightarrow \mathrm{ZZ}$ can be measured with 10-20\% accuracy for $m_{\mathrm{H}}>125 \mathrm{GeV}$. The Higgs couplings to Z, W, $\tau$ and top-quark can be measured with an accuracy of better than $20 \%$, for Higgs bosons heavier than $150 \mathrm{GeV}$.

\section{$5 \quad$ Search for Minimal Supersymmetric Standard Model Higgs bosons}

The prospects for the detection of the MSSM Higgs bosons at LHC have been evaluated $[9,12]$ in the scenario in which supersymmetric (SUSY) particle masses are large, so that the Higgs bosons do not decay in SUSY particles. At three level, all Higgs boson masses and couplings can be expressed in terms of only two parameters: $m_{\mathrm{A}}$, the mass of the CP-odd boson, and $\tan \beta$, the ratio of the vacuum expectation values of the two Higgs doublets. Some of the SUSY Higgs decay modes are the same as for the SM Higgs boson: $h \rightarrow \gamma \gamma$, tth $\rightarrow$ ttb $\bar{b}$.

Others modes, strongly enhanced at high $\tan \beta$, e.g. $\mathrm{H} / \mathrm{A} \rightarrow \tau^{+} \tau^{-}$, $\mathrm{H} / \mathrm{A} \rightarrow \mu^{+} \mu^{-}$, have been investigated [9]. The conclusion of these studies is that the complete region of parameter space should be accessible for the discovery of MSSM Higgs bosons, already with an integrated luminosity $\int \mathcal{L} \mathrm{dt}=30$ $\mathrm{fb}^{-1}$. Over a large fraction of the parameter space, more than one Higgs boson and more than one decay mode can be detected. An exhaustive summary of 

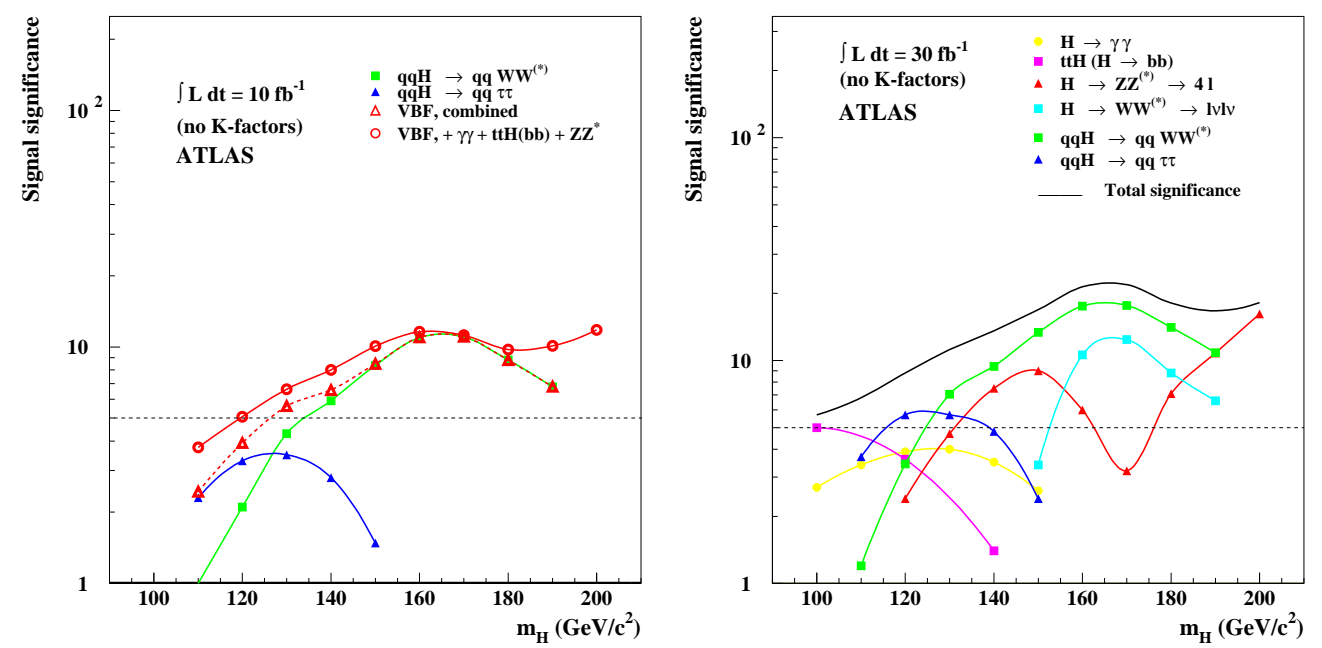

Figure 5: ATLAS sensitivity for the discovery of a Standard Model Higgs Boson for $\int \mathcal{L} \mathrm{dt}=10$ $\mathrm{fb}^{-1}$ (left), and $\int \mathcal{L} \mathrm{dt}=30 \mathrm{fb}^{-1}$ (right).

accessible decay modes can be found in Ref. [9]. The most difficult region is at moderate $\tan \beta$ and large $m_{\mathrm{A}}$, where only the lightest Higgs boson would be observable.

As in the SM case the search for Higgs boson decay in $\gamma \gamma$ can also be performed using associated $\mathrm{WH}$ and ttH production. The sensitivity of these channels is shown in Fig. 6a).

From Fig. 6a) it is clear that the region with $m_{\mathrm{h}}$ approximately $100 \mathrm{GeV}$ and $\tan \beta$ greater than 10 is not covered by the above mentioned decay channels of the $\mathrm{h}$ boson. In the most general case, considering all MSSM Higgs bosons, this region is accessible to the neutral boson $\mathrm{H}$ decays into $\mu^{+} \mu^{-}, \tau^{+} \tau^{-}$and to charged boson decays $\mathrm{H}^{ \pm} \rightarrow \tau \nu$. Recent studies have been performed to explore this region using the channel $\mathrm{b} \overline{\mathrm{b}} \mathrm{h} \rightarrow \mu^{+} \mu^{-}$(Fig. 6b) [14,15]. This search requires at the same time an excellent performance in $\mu$ detection and in b-tagging. Both features are offered by ATLAS.

The contributions of all channels investigated by ATLAS and CMS are summarized in Fig. 7 as $5 \sigma$ contours in the $m_{\mathrm{A}}$ vs. $\tan \beta$ plane. 

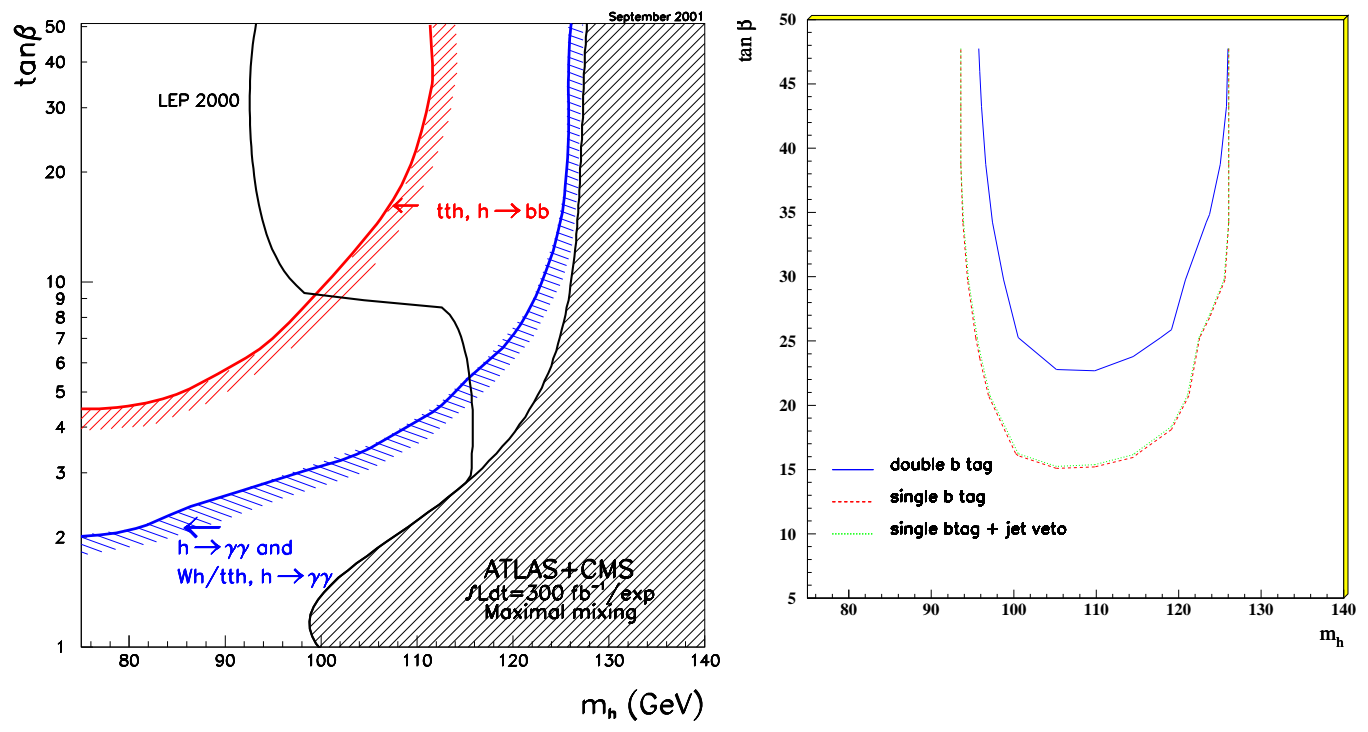

Figure 6: a) The ATLAS+CMS sensitivity for the discovery of the MSSM Higgs boson $\mathrm{h}$, in case of maximal mixing. The $5 \sigma$ discovery contour curves are shown in the $\left(\tan \beta, \mathrm{m}_{\mathrm{h}}\right)$ plane for the individual channels and for an integrated luminosity of $300 \mathrm{fb}^{-1}$. The LEP limits are also shown. The forbidden region is hatched [13]. b) Plot of discovery potential for the bbh channel with $\mathrm{h} \rightarrow \mu^{+} \mu^{-}$in the maximal mixing scenario. The curves correspond to a signal significance $\frac{s}{\sqrt{B}}=5$. The integrated luminosity is $\int \mathcal{L} \mathrm{dt}=300 \mathrm{fb}^{-1}$.

\section{Conclusions}

The LHC discovery potential for the Standard Model Higgs and MSSM Higgs bosons are extremely promising already in the first year(s) of data taking if the total integrated luminosity is in the range $10-30 \mathrm{fb}^{-1}$. Measurements of Higgs parameters can reach a precision of $0.1-1.0 \%$ for the mass determination, $5-30 \%$ for the branching ratios and $10-40 \%$ for the couplings with $\int \mathcal{L} \mathrm{dt}=300$ $\mathrm{fb}^{-1}$.

\section{Acknowledgments}

I would like to thank Elzbieta Richter-Was for her useful comments and Fabiola Gianotti for the helpful discussion, detailed comments and the critical reading of manuscript. 


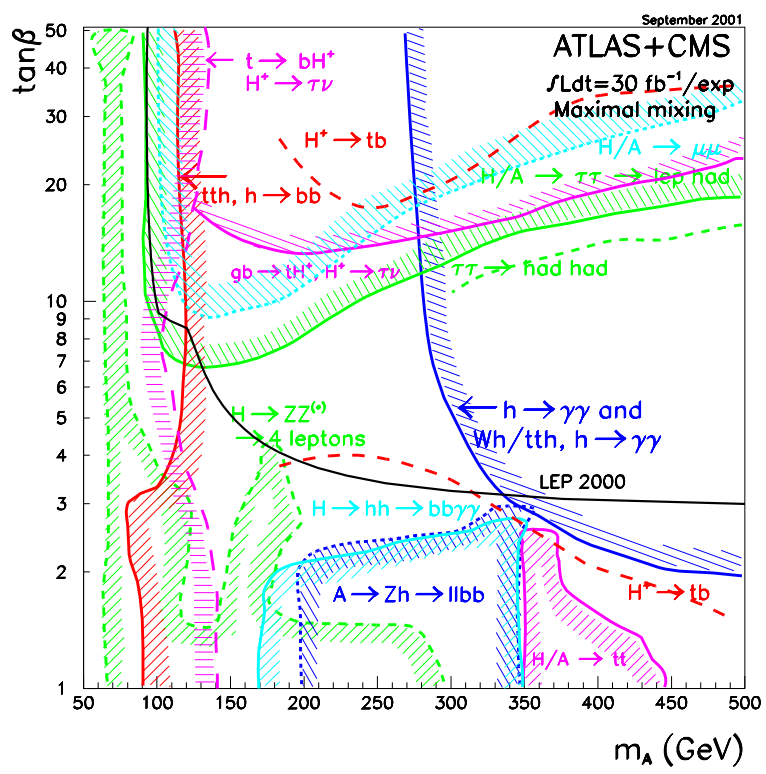

Figure 7: The ATLAS+CMS sensitivity for the discovery of the MSSM Higgs bosons, in the case of maximal mixing. The $5 \sigma$ discovery curves are shown in the $\left(\tan \beta, \mathrm{m}_{\mathrm{A}}\right)$ plane for the individual channels and for an integrated luminosity of $30 \mathrm{fb}^{-1}$. The LEP limit is also shown.

\section{References}

[1] S.L. Glashow, Nucl.Phys. 22, 579 (1961); S.Weinberg,, Phys.Rev.Lett 19, 1264 (1967); A.Salam," Elementary Particle theory",Ed.N. Svarthlom (Altmqvist ad Wiksells, Stockolm) (1968), 367.

[2] The ALEPH and DELPHI and L3 and and OPAL Collaborations and the LEP Elecroweak working group, CERN-EP/2002-091.

[3] ALEPH, DELPHI, L3, OPAL, The LEP Working Group For Higgs Boson Search Phys.Lett. B 565, 61 (2003)

[4] H.-P. Nilles, Phys.Rep. 1 (1984); H. E. Haber and G. L. Kane, Phys.Rep. 75 (1985); R. Barbieri,Riv. Nuovo. Cim. (1988) 11.

[5] The ALEPH and DELPHI and L3 and and OPAL Collaborations and the LEP Higgs working group, LHWG Note 2001-05 (2001).

[6] The ALEPH and DELPHI and L3 and and OPAL Collaborations and the LEP Higgs working group, LHWG Note 2001-04 (2001).

[7] The CDF and and D0 Collaborations and the Tevatron Higgs Sensitivity group, FERMILAB-PUB-03/320-E (2003) 
[8] CMS Coll., Tecnical Proposal, report CERN/LHCC/94-38 (1994).

[9] ATLAS Coll., ATLAS Detector and Physics Performance:

Technical Design Report,Volume 2, report CERN/LHCC/99-15 (1999).

[10] S.Asai et al.,Prospect for search of a Standard Model Higgs Boson in ATLAS using Vector Boson Fusion, SN-ATLAS-2003-024.(2003)

[11] M. Duehrssen,Prospects for the measurement of Higgs boson coupling parameters in the mass rang 110-190 GeV, ATL-PHYS-2003-030 (2003).

[12] E. Richter-Was et al., J.Mod.Phys. 1371 (1998).

[13] F. Gianotti, Presentation at LHC Committee 5 July 2000.

[14] S.Gentile et al. Search for supersymmetric neutral Higgs $h$ in the decay $h \rightarrow \mu^{+} \mu^{-}$in ATLAS detector at LHC, ATL-PHYS-2003-013,(2003).

[15] S.Gonzales et al. Analysis of the process $p p \rightarrow \mathrm{b} \overline{\mathrm{b}} h / A \rightarrow \mu^{+} \mu^{-}$in MSSM with $m_{\mathrm{A}}$ i125 GeV, ATL-PHYS-2002-021,(2002). 